\title{
Foveal warning stimuli and predictive saccades to a constant-location target
}

\author{
LEONARD E. ROSS and SUSAN M. ROSS \\ University of Wisconsin, Madison, Wisconsin 53706
}

\begin{abstract}
The frequency of predictive saccades to a constant-location target was investigated as a function of a number of foveal warning stimulus conditions. The offset of a fixation stimulus led to a high level of predictive responding, whereas much lower response levels were found with other warning conditions, including the onset or offset of a stimulus in a defined fixation area and the onset of a target without defining fixation lines. It was concluded that the continuing presence of a foveal stimulus during the warning interval resulted in little predictive responding. Various interpretations of this finding are discussed.
\end{abstract}

This study investigated the effects of various foveal warning stimulus conditions on predictive saccades made to targets presented at a constant location. Predictive saccades (i.e., saccades that anticipate the occurrence of a target at a predictable location) usually have been investigated in square-wave tracking situations in which latency of the saccade relative to the movement of the target provides a measure of the saccade's anticipation of target movement. A number of investigators (e.g., see Stark, 1971) who have used such square-wave targets have reported saccade response delays less than the response latency of saccadic eye movements to unpredictable targets and, in some cases, have found eye movements that anticipated the target movement. One study (Dallos \& Jones, 1963) that investigated the acquisition of such predictive responding found that the time lag of saccades to square-wave stimuli decreased gradually over a period of up to five cycles.

The present study examined predictive saccadic eye movements under conditions in which a warning event reliably predicted the occurrence of a peripheral target at a fixed location. The study was designed to investigate predictive responding as a function of the onset vs. the offset of a foveal warning stimulus and the presence or absence of fixation stimuli during the interval from the onset of the warning event until the onset of the target. Interest in the warning signal onsetoffset factor stemmed from data (Ross \& Ross, 1980) indicating that a stimulus-offset warning event was more effective than either stimulus onset or stimulus change in reducing saccade latency to unpredictable targets. In addition, when a warning event was presented 100 $150 \mathrm{msec}$ following the target, saccade latency was longer with stimulus onset than with either stimulus

This study was supported by United States Public Health Service Grant HD 08240. Requests for reprints should be sent to L. E. Ross, Department of Psychology, University of Wisconsin, Madison, Wisconsin 53706. offset or a no-warning control condition. These results demonstrated that onset and offset warning events could have quite different effects on the latency of saccades to unpredictable targets, which raised the possibility that similar differences might also be found with respect to their effects on predictive saccades to constantlocation targets.

Four groups were included in the present study. Two of the groups were used to compare the effectiveness of stimulus onset and offset as warning events for predictive saccades. For a stimulus-onset group, a warning stimulus appeared at the fixation point $800 \mathrm{msec}$ prior to the occurrence of the target and remained on until target offset. For a corresponding stimulus-offset group, the foveal stimulus remained on at all times except for the interval from $800 \mathrm{msec}$ prior to the target onset until target offset. In order to establish a fixation area for the subjects of both groups, the onset and offset warning stimuli appeared in an area defined by four diagonal lines that remained on at all times. In addition to these onset and offset conditions that employed fixation lines, two additional groups were included to determine the relative effects of having a foveal stimulus remain or not remain after the occurrence of a warning event. For one group, the offset of a fixation stimulus served as the warning event, a procedure that has been employed in a number of studies investigating the effects of warning on saccade latency and was also the condition that led to the shortest saccade latency to unpredictable targets in the Ross and Ross (1980) study. Another group was tested under conditions identical to those of the fixation lines onset group, except that the fixation lines terminated at the onset of the warning stimulus and reappeared at the time of target offset. This condition was included to investigate the possibility that the presence of fixation lines might reduce predictive responding by inducing some form of implicit instructions not to make eye movements away from the location defined by the lines as they remained during the warning interval. 


\section{METHOD}

\section{Subjects}

Subjects were 32 undergraduate students $(16$ males and 16 females) enrolled in introductory psychology courses at the University of Wisconsin. All had 20/20 uncorrected near vision in both eyes as measured by a Titmus Vision-Tester.

\section{Apparatus}

The experiment was conducted in a two-room laboratory suite. The subject room was divided by a black curtain extending from floor to ceiling. A portion of the Hewlett-Packard Model 1321A display oscilloscope with a fast (P15) phosphor was visible through a $2.5 \times 36.5 \mathrm{~cm}$ slot in a black panel located in the plane of the curtain. All stimuli presented on the screen had an intensity of 1 nit. The subject was seated in an ophthalmological examination chair with the headrest positioned so that the slot in the cardboard panel was at the subject's eye level at a viewing distance of $60 \mathrm{~cm}$. The subject room was dark during the experiment except for a single 15-W incandescent bulb that provided indirect lighting.

The stimulus generating and response recording equipment was located in an adjacent control room, with an intercom system used to permit communication between the experimenter and the subject. The stimuli presented on the display screen were generated and timed by a laboratory computer.

The subject's eye movements were detected using a Narco Biometric Eye Trac Model 200 eye movement monitor. Horizontal eye movements were recorded from the right eye, and vertical eye movements were recorded from the left eye. During each trial, the unfiltered Eye Trac output for the right eye (horizontal eye movements) was sampled every $5 \mathrm{msec}$ and digitized by a laboratory computer that also identified the onset of the first saccade and determined its latency. At the conclusion of the trial, conditions and saccade latency were printed, and the saccade, with the computer-determined saccade onset identified, was displayed on a monitor scope for the experimenter's inspection. Permanent records of the unfiltered analog output of the Eye Trac were made using a Beckman R511A dynograph.

\section{Procedure}

The subjects were randomly assigned to the four groups of eight subjects each, with the restriction that each group include four females and four males. All subjects received 60 trials with the target, the letter X, appearing 15 deg to the left of the fixation point for half of the subjects and $15 \mathrm{deg}$ to the right for the other half. The X subtended visual angels of .5 deg vertically and $.35 \mathrm{deg}$ horizontally and had a duration of $1 \mathrm{sec}$. For three of the groups, a fixation pattern was used that consisted of four diagonal lines, each subtending $.43 \mathrm{deg}$ radiating out at $45 \mathrm{deg}$ from the four corners of an imaginary square that subtended $1.32 \mathrm{deg}$ of visual angle on each side. The warning event for all groups was the onset or offset of the letter $\mathrm{O}$, which subtended $.5 \mathrm{deg}$ vertically and $.35 \mathrm{deg}$ horizontally.

For one group (no-lines/offset) the letter $O$ was present as the fixation point until $800 \mathrm{msec}$ prior to target onset, when its offset served as the warning event. The $O$ reappeared at the center of the screen at the time of target offset. This condition, in which the offset of the fixation stimulus served as the warning event, is similar to those employed in most of the studies investigating the effects of a warning signal on saccade latency to an unpredictable target.

For two groups (lines-onset and lines-offset), the onset or offset of the letter $\mathrm{O}$ served as the warning event. Thus for the lines-onset group, the letter O's onset $800 \mathrm{msec}$ prior to target onset served as the warning event, whereas for the lines-offset group, the letter O's offset $800 \mathrm{msec}$ prior to the target onset was the warning event. The four-line fixation pattern was present on the screen continuously throughout the trials for these two groups.

The final group (lines-off/onset) was identical to the linesonset group except that the fixation lines went off at warning signal onset and remained off until target offset.

Upon arriving at the laboratory, all subjects received a vision test and were then seated in front of the display screen and told that from time to time the letter $\mathrm{X}$ would appear somewhere in the narrow window. The fixation stimulus was pointed out, and the subject was instructed to look at the $\mathrm{X}$ as quickly as possible each time that it appeared. The subjects were asked to sit still, but they were encouraged to blink their eyes normally. The sensors were then fitted to the subject and adjusted as necessary. The lights in the subject room were then turned off, except for the 15-W lamp. After a 2 -min period, the eye movement monitoring equipment was calibrated by having the subject look at various numbers in a horizontal five-number array. This procedure took about $5 \mathrm{~min}$, and trials began approximately 2 min after the disappearance of the calibration array.

Immediately prior to each trial, the subject's eye position was checked by the computer. If the subject's gaze was not within $1 \mathrm{deg}$ of the center of the fixation area, or if the subject's eyes were closed, the trial was aborted and attempted again approximately $3 \mathrm{sec}$ later. If the subject's gaze still was not within the fixation area, trials were halted briefly, and, if necessary, the Eye Trac sensors were recentered to compensate for changes in the subject's head position. Following each trial, the experimenter examined the tracing on the monitor oscilloscope and initiated the next trial. This was followed by a computercontrolled variable pause of 4,8 , or 12 sec.

\section{RESULTS}

Pilot data collected under conditions in which a target appeared at a predictable location but was not preceded by a warning stimulus showed that the latency of a saccade to this type of target was at least $110 \mathrm{msec}$. Predictive saccades were therefore defined as those occurring within the interval from the onset of the warning event to $100 \mathrm{msec}$ after target onset.

Figure 1 shows the average frequencies of predictive saccades, in blocks of 10 trials, for the four groups. It can be seen that the no-lines/offset group showed a gradual increase in frequency of predictive saccades over trial blocks and reached a much higher level of responding than did the other three groups. These observations are confirmed by the results of an analysis of variance with groups, sex of subject, and target direction as between-subjects factors and trial blocks as a withinsubjects factor. There was a significant main effect of trial blocks $[\mathrm{F}(5,80)=17.40, \mathrm{p}<.001]$, and although the main effect of groups was not significant $[\mathrm{F}(3,16)=$ 1.49], there was a significant interaction between groups and trial blocks $[F(15,80)=2.00, p<.05]$.

In order to evaluate this interaction, individual analysis of variance, including target direction, sex of subject, and trial blocks, were carried out for each of the four groups. These analyses resulted in significant main effects of trial blocks for the no-lines/offset and linesoffset groups $[F(5,20)=12.23$ and $5.89, p<.001$ and .005 , respectively], but not for the lines-onset and lines-off/onset groups $[F(5,20)=1.19$ and 2.50 , respectively]. Subsequent $t$ tests indicated significant increases 


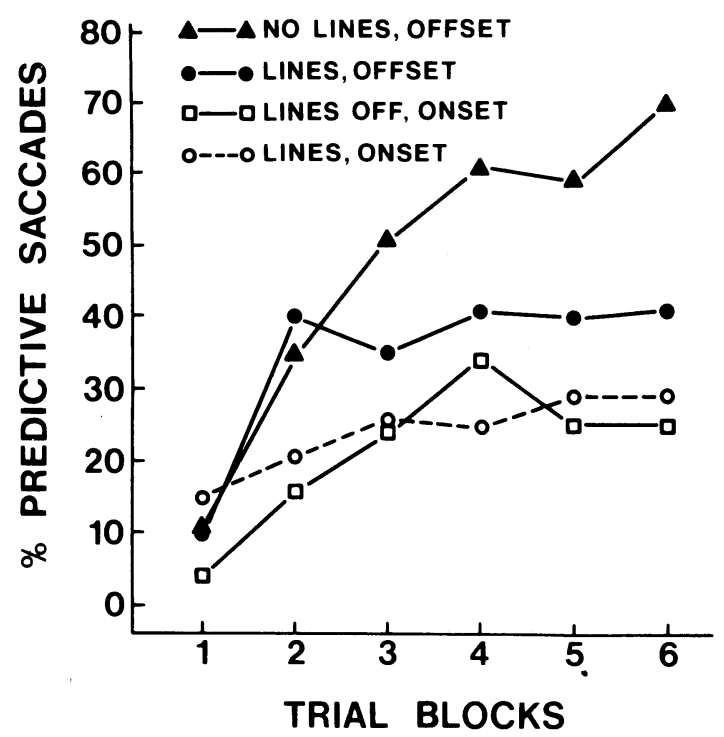

Figure 1. Percentage predictive saccadic responses as a function of blocks of 10 trials. The lines, no-lines, and lines-off notation refers to the presence, absence, or offset of four fixation lines; onset and offset refer to whether the warning event was a stimulus onset or offset.

between Blocks 1 and 2 and between Blocks 2 and 3 for the no-lines/offset group [ $\mathrm{t}(80)=2.98$ and $2.04, \mathrm{p}<.001$ and .05 , respectively] and a significant increase in anticipatory responding between Blocks 1 and 2 for the lines-offset group $[t(80)=3.77, p<.001]$.

Separate analyses of variance were also carried out to compare the four groups on the fifth trial block and the sixth block. Both analyses resulted in a significant main effect of groups $[F(3,80)=7.24$ and $13.45, p<.001$, for Block 5 and Block 6 , respectively]. Subsequent $t$ tests indicated that for both blocks, the no-lines/offset group gave significantly more predictive saccades than did the other three groups $[\mathrm{t}(80)=2.35,3.77$, and 4.24 for Block 5 and $t(80)=3.61,5.34$, and 5.65 for Block 6]. The three groups for which a stimulus remained on the screen during the warning interval were not significantly different in their final levels of predictive responding.

\section{DISCUSSION}

The only groups that showed a significant increase in predictive saccades were those in which warning signal offset served as the warning event (i.e., the no-lines/offset and the lines-offset groups). In the other conditions, in which either the fixation lines and the warning signal (lines-onset group) or the warning signal alone (lines-off/onset group) remained present during the warning interval, predictive responding remained at a low level. While these groups did show a small increase of slightly less than $20 \%$ in predictive responding, the increase was not statistically significant.

The fact that there was a significant increase in responding on the part of the lines-offset group but not the lines-onset group suggests that the former condition was somewhat more effective than the latter in leading to the occurrence of predic- tive saccades, although the difference in absolute level of responding between the two groups was not significant. An onset-offset difference would be consistent with the data of Ross and Ross (1980) that showed that a stimulus-offset warning event had a greater effect on the latency of saccades to an unpredictable target than did stimulus onset. Another similarity between the effects of warning events on the latency of saccades to unpredictable targets and on the level of predictive responding is the fact that the greatest response facilitation occurred with the no-lines/offset condition in both the Ross and Ross (1980) study and the present experiment.

There are several possible interpretations of the high level of predictive responding displayed by the notines/offset group. First, it is possible that the offset of all stimuli served as some kind of "releaser," in that the eye may have a tendency to remain fixed at a location as long as a stimulus that has functional value is in foveal view. In the case of the onset warning groups, the onset of the warning stimulus could have been such a stimulus. Perhaps the suggestion of Potter and Levy (1969), that the eye remains relatively fixed at the place information is rapidly being presented in order to process the changing information, applies in such situations.

However, even when the warning event was a stimulus offset, the level of predictive responding was greatly depressed when the fixation lines remained, as is seen by a comparison of the nolines/offset and the lines-offset groups. This result indicates that stimulus change is not necessary for a foveal stimulus, in this case the remaining fixation lines, to reduce the tendency of the eye to move in anticipation of the appearance of the target. This raises the possibility that the lower level of predictive responding found with a continuing foveal stimulus reflects some instructional set factor. Thus the occurrence of a predictive saccade could represent a violation of implicit instruction, induced by the presence of foveal stimuli, to continue to fixate the fixation area during the warning interval. While this type of factor could have affected the present data, there are several considerations that reduce the likelihood that it can provide a complete account of these findings. First, the lines-off/onset group showed a very low level of predictive responding, which demonstrates that any instructional set not to respond would have to be elicited not only by the presence of the stimuli defining the fixation area, but also by the continued presence of the warning signal with the fixation lines absent. Second, the correspondence that exists between the effects of various foveal stimulus conditions on predictive responding in the present study and the latency of nonpredictive responding to unpredictable targets as found in the Ross and Ross (1980) data suggests that the mechanism is not primarily one of the violation of implicit instructions, since in the latter case the saccades did not occur prior to the occurrence of the target. Third, in an earlier unreported study eight subjects were tested in a predictive responding situation that also compared stimulus offset, the offset of the fixation stimulus, and stimulus onset, the change of the fixation stimulus to a new shape, but did so with the comparison as a within-subjects variable. The predictive responding on fixation-offset trials showed a gradual increase to approximately $75 \%$, whereas predictive responding on fixation-change trials remained at approximately $30 \%$; these response levels are comparable to those of the corresponding no-lines/offset and lines-off/onset groups of the present study. These results show that the effects of foveal stimulus presence or absence on predictive responding can appear on a trial-by-trial basis and thus are unlikely to reflect a general attitude or set not to respond prior to the onset of the target.

Thus while it is possible that some sort of implicit instructions or set effects could play a role in the effect of foveal stimuli on predictive saccadic responding, the above considerations suggest that other mechanisms, probably related to the effects of foveal stimuli on information intake or saccadic programming processes, are of primary importance. 


\section{REFERENCES}

Dallos, P. J., \& Jones, R. W. Learning behavior of the eye fixation control system. IEEE Transactions on Automatic Control, 1963, AC-8, 218-227.

Potter, M. C., \& Levy, E. I. Recognition memory for a rapid sequence of pictures. Journal of Experimental Psychology, $1969,81,10-15$.
Ross, L. E., \& Ross, S. M. Saccade latency and warning signals: Stimulus onset, offset, and change as warning events. Perception \& Psychophysics, 1980, 27, 251-257.

StARK, L. The control of eye movements. In P. Bach-y-Rita, C. C. Collins, \& J. E. Hyde (Eds.), The control of eye movements. New York: Academic Press, 1971.

(Received for publication October 30, 1980.) 